\title{
Parallel and symmetric 2-tensor fields on pseudo-Riemannian cones
}

\author{
PierRe Mounoud
}

\begin{abstract}
We study the complete pseudo-Riemannian manifolds whose cone admits a non-trivial parallel symmetric 2-tensor field. We are able to give an extensive description of these manifolds except when the tensor field is given by a nilpotent endomorphism. In this case, we are able to describe only a dense open subset of them. Moreover, we construct examples with non-constant curvature where this open set is proper.
\end{abstract}

\section{Introduction}

Let $(M, g)$ be a pseudo-Riemannian manifold (we will consider that a Riemannian metric is also a pseudo-Riemannian one). It is interesting to associate to $(M, g)$ its cone $(\widehat{M}, \widehat{g})$ defined by $\widehat{M}=\mathbb{R}_{>0} \times M$ and $\widehat{g}=d r^{2}+$ $r^{2} g$. This construction appears in different contexts. For example (pseudo-) Sasakian manifolds are characterized by the fact that their cone is (pseudo-) Kähler (see [3]). Cones are also used by Bär in [2] to classify Riemannian manifolds admitting a non-trivial Killing spinor: indeed a Riemannian manifold admits a real Killing spinor if and only if its cone admits a parallel spinor (see [4] for the pseudo-Riemannian analogue). In [5], Gallot used cones to study the Obata equation. This situation led Alekseevski et al. to study in [1] the holonomy of cones as a subject on its own.

In this paper we are interested in describing the pseudo-Riemannian manifolds $(M, g)$ whose cone has a non-trivial parallel symmetric 2-tensor. As such it can be seen as a contribution to the study of the holonomy of cones, in the same spirit as [1]. Indeed most of our results extend, more or less directly, results of [1]. Besides, Gallot proved in [5] (see also [7]) that there is a one-to-one correspondence (recalled in Section 2.1) between these tensors and the solutions of the Obata equation of $(M, g)$. We recall that a function $\alpha$ is a solution of the Obata equation if, for any tangent vector $u$, 
$v$ and $w$,

$$
\begin{aligned}
& D D D \alpha(u, v, w)+2(D \alpha \otimes g)(u, v, w)+(D \alpha \otimes g)(v, u, w) \\
& \quad+(D \alpha \otimes g)(w, u, v)=0,
\end{aligned}
$$

where $D$ is the Levi-Civita connection of $g$.

The interest of Gallot in this equation came from spectral geometry as an eigenfunction for the third eigenvalue of the Laplacian of the sphere is a solution of this equation. But this equation is also present in projective geometry: it appears in the work of Solodovnikov [10] (see also the work of Kiosak and Matveev [6] in the pseudo-Riemannian case) where it is related to the existence of projectively equivalent metrics, i.e., metrics having the same unparameterized geodesics.

Gallot and Tanno have independently shown (see $[5,11])$ that if $(M, g)$ is a complete Riemannian manifold admitting a non-constant solution to the Obata equation then $(M, g)$ is a quotient of the round sphere. This result has been extended to the pseudo-Riemannian case, keeping the conclusion, by Matveev and the author in [7] under the hypothesis that $(M, g)$ is compact but not necessarily geodesically complete.

Hence, this work is also a way to obtain a further generalization of the Gallot-Tanno theorem. As we are interested in global results, we need an hypothesis of maximality on $(M, g)$. Ideally, we would like to assume only that $(M, g)$ is non-extendable (i.e., that it is not isometric to a proper open subset of any other manifold). Unfortunately, this is not clear whether this hypothesis is sufficient. Consequently, we will use the original hypothesis of geodesical completeness. But, as in the pseudo-Riemannian context this hypothesis is quite restrictive, we will also consider the alternative hypothesis that there exists a solution $\alpha$ of the Obata equation which is proper, i.e., such that for any compact $K \subset \mathbb{R}, \alpha^{-1}(K)$ is compact.

From now on, all manifolds will be assumed to be connected. Let us consider a parallel tensor field $T$ on $\widehat{M}$ and $\widetilde{T}$ the endomorphism associated to $T$ (i.e., we have $T(u, v)=\widehat{g}(u, \widetilde{T}(v)))$. If $T$ is not trivial, i.e., not proportional to $\widehat{g}$, we can assume (see Proposition 2.6) that:

- either $\widetilde{T}^{2}=\widetilde{T}$ which means that $(\widehat{M}, \widehat{g})$ is decomposable, i.e., $\widehat{M}$ admits a non-degenerate proper parallel distribution or equivalently that $(\widehat{M}, \widehat{g})$ is locally a product,

- either $\widetilde{T}^{2}=-\mathrm{Id}$, in this case $(\widehat{M}, \widehat{g}, T)$ defines a complex Riemannian structure, i.e., $T$ defines a complex structure on $\widehat{M}$ and $\widehat{g}-i T$ defines a field of non-degenerate complex bilinear forms on $\widehat{M}$, 
- or $\widetilde{T}^{2}=0$, i.e., $\widetilde{T}$ is 2 -step nilpotent, in this case (as far as the author knows) there is no classical geometric interpretation.

It turns out that the simplest case is the complex Riemannian one. In this situation, we are able to describe the whole manifold and therefore a large family of manifolds admitting non-trivial solutions to the Obata equation.

Theorem 1.1. Let $(M, g)$ be a non-extendable connected pseudo-Riemannian manifold having a complex Riemannian cone $(\widehat{M}, \widehat{g}, T)$. Let $\alpha$ be the function on $M$ associated to $T$. If $(M, g)$ is complete or if $\alpha$ has compact levels then there exists a complex Riemannian manifold $(N, h, S)$ such that $M=\mathbb{R} \times N$ and

$$
g=-d s^{2}+h-\sinh (2 s) S .
$$

Conversely, the cone over such a manifold admits a complex Riemannian structure.

We are also able to classify, under any of our global hypotheses on $(M, g, \alpha)$, the pseudo-Riemannian manifolds with decomposable cone. This result extends Theorem 7.1 from [1] and can be stated as follows:

Theorem 1.2. Let $(M, g)$ be a connected pseudo-Riemannian manifold with decomposable cone. If $g$ is complete or if the function $\alpha$ associated to the cone decomposition is proper then

- either $g$ has constant curvature equal to 1 ,

- or $\widetilde{M}$, the universal cover of $M$, is a warped product of a negative definite hyperbolic space (possibly 1-dimensional) and a pseudo-Riemannian manifold $\left(F, g_{F}\right)$.

More precisely, using polar coordinates on the hyperbolic space the metric reads $g=-d s^{2}-\sinh ^{2}(s) g_{S}+\cosh ^{2}(s) g_{F}$, where $g_{S}$ is the standard metric of the sphere.

More precisions about the topology of $M$ are given in Theorem 4.11.

The last situation corresponds to cones admitting a parallel nilpotent symmetric endomorphism field. In this case, we obtain a description of an open dense subset of $(M, g)$. This is a generalization of Theorem 9.1 of [1], which corresponds to the case where the rank of $\widetilde{T}$ is 1 .

Theorem 1.3. Let $(M, g)$ be a non-extendable pseudo-Riemannian manifold such that $(\widehat{M}, \widehat{g})$ has a non-trivial parallel symmetric 2-tensor field $T$ 
such that $\widetilde{T}^{2}=0$. Let $\alpha$ be the function associated to $T$. Then $M \backslash \alpha^{-1}(0)$ is dense; let $U$ be one of its connected component.

If $(M, g)$ is complete or if $\alpha$ has compact levels then there exists a pseudoRiemannian manifold $(N, h)$ endowed with a parallel symmetric and nilpotent 2-tensor field $S$ such that $U=\mathbb{R} \times N$ and

$$
g=-d s^{2}+e^{2 s}(h-S)+S .
$$

Unfortunately, we have not been able to describe the whole manifold in this case. But we provide in Section 5 a family of pseudo-Riemannian manifolds with non-constant curvature having a cone endowed with a nilpotent parallel endomorphism field and such that $\alpha^{-1}(0)$ is not empty. These metrics are perturbations of the pseudo-sphere $S^{p, q}=\left\{x \in \mathbb{R}^{p+1, q} \mid\langle x, x\rangle=1\right\}$, where $\mathbb{R}^{p+1, q}$ stands for $\mathbb{R}^{p+q+1}$ endowed with a quadratic form of signature $(p+1, q)$. It is our opinion that, even if it is not clear that our examples are complete, this construction shows a lack of rigidity that explains why the classification is more complicated in this case.

We conclude this paper by explicating how under certain conditions a parallel 2-tensor on $\widehat{M}$ provides projectively equivalent metrics on $M$.

The author wishes to thank the referees for their suggestions and comments in order to improve this article.

\section{Parallel symmetric 2-tensors on the cone over a manifold}

\subsection{Link with the Obata equation}

We start by giving the definition of cones over pseudo-Riemannian manifolds.

Definition 2.1. Let $(M, g)$ be a pseudo-Riemannian manifold. We call cone manifold over $(M, g)$ the manifold $\widehat{M}=\mathbb{R}_{>0} \times M$ endowed with the metric $\widehat{g}$ defined by $\widehat{g}=d r^{2}+r^{2} g$.

We will denote by $D$ the Levi-Civita connection of $g$ and by $\widehat{D}$ the Levi-Civita connection of $\widehat{g}$. Those connections are related by the following fact.

Fact 2.2. The Levi-Civita connection of $\widehat{g}$ is given by

$$
\widehat{D}_{X} Y=D_{X} Y-r g(X, Y) \partial_{r}, \quad \widehat{D}_{\partial_{r}} \partial_{r}=0, \quad \widehat{D}_{\partial_{r}} X=\widehat{D}_{X} \partial_{r}=\frac{1}{r} X .
$$


The holonomy of cones over pseudo-Riemannian manifolds is strongly related to the Obata equation seen in the introduction. This relation is given by the following proposition, which is proved in [7] by following the lines of $[5]$.

Proposition 2.3 (see [7], Proposition 3.1). Let $(M, g)$ be a pseudoRiemannian manifold. Let $(\widehat{M}, \widehat{g})$ be the cone manifold over $(M, g)$. There exists a smooth non-constant function $\alpha: M \rightarrow \mathbb{R}$ such that for any vectors $u, v, w$ tangent to $M$ we have:

$$
\begin{aligned}
& D D D \alpha(u, v, w)+2(D \alpha \otimes g)(u, v, w)+(D \alpha \otimes g)(v, u, w) \\
& \quad+(D \alpha \otimes g)(w, u, v)=0
\end{aligned}
$$

if and only if there exists a non-trivial symmetric parallel 2-tensor field on $(\widehat{M}, \widehat{g})$.

More precisely if $\alpha$ is a non-trivial solution of the above equation then the Hessian of the function $A: \widehat{M} \rightarrow \mathbb{R}$ defined by $A(r, m)=r^{2} \alpha(m)$ is parallel (i.e., $\widehat{D} \widehat{D} \widehat{D} A=0)$. Conversely if $T$ is a symmetric parallel 2-tensor field on $\widehat{M}$ then $T\left(\partial_{r}, \partial_{r}\right)$ does not depend on $r$ and is a solution of the above equation. Moreover, $2 T$ is the Hessian of the function $A_{T}$ defined by $A_{T}(r, m)=r^{2} T_{(r, m)}\left(\partial_{r}, \partial_{r}\right)$.

We just quote the following Lemma, it is one of the steps of the proof of Proposition 2.3 and it will be useful further.

Lemma 2.4 ([7], Corollary 3.3). Let $T$ be a symmetric parallel 2-tensorfield on $(\widehat{M}, \widehat{g})$, and let $\alpha=T\left(\partial_{r}, \partial_{r}\right)$. Let $u, v, w$ be vectors tangent to $M$ also seen as vectors perpendicular to $\partial_{r}$ in $\widehat{M}$. We have

$$
\begin{aligned}
2 T\left(\partial_{r}, u\right) & =r D \alpha(u), \\
2 T(u, v) & =r^{2}(2 g(u, v) \alpha+D D \alpha(u, v)), \\
2 D T(u, v, w) & =-D \alpha \otimes g(v, u, w)-D \alpha \otimes g(w, u, v) .
\end{aligned}
$$

Example 2.5. It follows from Fact 2.2, that the curvature of $\widehat{g}$ is given by

$$
\widehat{R}(u, v) w=R(u, v) w-g(v, w) u+g(u, w) v,
$$

where $R$ and $\widehat{R}$ are the curvature tensors of $g$ and $\widehat{g}$. Thus, a simply connected pseudo-Riemannian manifold $(M, g)$ with constant curvature equal to 1 has a cone, which is flat and simply connected. Hence the cone over $(M, g)$ 
admits any kind of parallel tensor fields. This is somehow a trivial example. We are going to look for non-trivial ones.

Contrarily to the Riemannian case, the existence of a parallel symmetric 2-tensor on a pseudo-Riemannian manifold does not imply that the manifold is decomposable (i.e., it possesses parallel non-degenerate distributions). But the following proposition shows that it suffices to consider only three cases.

Proposition 2.6. If a pseudo-Riemannian manifold $(N, h)$ admits a nontrivial symmetric parallel endomorphism $\widetilde{T}$ then there exists on $(N, h)$ a symmetric parallel endomorphism $\widetilde{T}^{\prime}$ such that $\widetilde{T}^{\prime 2}=\widetilde{T}^{\prime}, \widetilde{T}^{\prime 2}=0$ or $\widetilde{T}^{\prime 2}=$ $-I d$.

Proof. If $(N, h)$ is decomposable, i.e., if there exists a non-degenerate parallel distribution $V$ on $(N, h)$ then the projection on $V$ is a parallel endomorphism $\widetilde{P}$ satisfying $\widetilde{P}^{2}=\widetilde{P}$. If $\widetilde{T}$ is a symmetric parallel endomorphism then it is also the case of its nilpotent part. If it is not trivial and if we take an appropriate power of it we obtain a non-trivial symmetric parallel endomorphism $\widetilde{T}^{\prime}$ such that $\widetilde{T}^{\prime 2}=0$.

At last, if $(N, h)$ is not decomposable and if $\widetilde{T}$ is a non-trivial semisimple symmetric parallel endomorphism then there exits $\lambda=a+i b \in \mathbb{C} \backslash \mathbb{R}$ such that the minimal polynomial of $\widetilde{T}$ is $(X-\lambda)(X-\bar{\lambda})$. But in this case $\widetilde{T}^{\prime}=\frac{1}{b} \widetilde{T}-\frac{a}{b}$ Id is parallel and symmetric and satisfies $\widetilde{T}^{\prime 2}=-\mathrm{Id}$.

Example 2.5 shows that any of these situations may occur on a cone over a complete pseudo-Riemannian manifold. However it is proven in [7] that on a cone over a compact manifold there is only one type of symmetric 2-tensor field to investigate:

Proposition 2.7 ([7], Proposition 3.4). Let $(M, g)$ be a closed pseudoRiemannian manifold. If the Obata equation has a non-constant solution then $(\widehat{M}, \widehat{g})$ is decomposable.

\subsection{Basic properties}

Let $(M, g)$ be a pseudo-Riemannian manifold such that its cone $(\widehat{M}, \widehat{g})$ admits a non-trivial symmetric parallel 2-tensor field $T$. Proposition 2.6 tells us that we can assume that its associated parallel endomorphism $\widetilde{T}$ satisfies $\widetilde{T}^{2}=\widetilde{T}, \widetilde{T}^{2}=0$ or $\widetilde{T}^{2}=-I d$. 
We recall that if $\lambda$ is an eigenvalue of $\widetilde{T}$ (here we can only have $\lambda=0$ or 1 ) then the eigenspace $V_{\lambda}$ associated to $\lambda$ is a parallel distribution and therefore is integrable, i.e., it defines a foliation.

As in Section 2, we define on $\widehat{M}$ the functions $\alpha=T\left(\partial_{r}, \partial_{r}\right)$ and $A$ by $A(r, m)=r^{2} \alpha(m)$. We recall that $\alpha$ is actually a function on $M$. We start with two corollaries of Proposition 2.3. The first one actually implies Proposition 2.7.

Corollary 2.8. The set of critical values of $\alpha$ is included in $\sigma(T)$, the spectrum of $\widetilde{T}$ (i.e., the set of its real eigenvalues).

Proof. Let $m \in M$ be a critical point of $\alpha$. Lemma 2.4 and Proposition 2.3 imply that for any $r>0$,

$$
\widehat{D} \widehat{D} A_{(r, m)}\left(\partial_{r}, .\right)=2 \alpha(m) \widehat{g}\left(\partial_{r}, .\right)
$$

It means that $\partial_{r}(r, m)$ belongs to the eigenspace of $\widetilde{T}$ associated to the eigenvalue $\alpha(m)$. In our cases it implies that $\alpha(m)=0$ or 1 .

Corollary 2.9. If $\alpha$ is constant on an open subset $U$ of $M$ then $\alpha$ is constant on $M$ and $T$ is trivial.

Proof. As for any $k \in \mathbb{R}$, the tensor field $T+k \widehat{g}$ is also parallel, we can assume that for any $m \in U, \alpha(m)=0$. Hence $A$ vanishes on $\mathbb{R}_{>0} \times U$ and so does the Hessian of $A$. But the Hessian of $A$ is equal to $2 T$ which is parallel. It means that $T$ is trivial and therefore $\alpha$ is constant.

We consider the vector field $Y$ on $\widehat{M}$ defined by

$$
Y=\widetilde{T}\left(\partial_{r}\right)
$$

We decompose now the vector field $Y$ according to the splitting $T \widehat{M}=$ $\mathbb{R} \partial_{r} \oplus T M$, we have

$$
Y=\alpha \partial_{r}+X
$$

where $X$ is a vector field on $\widehat{M}$ tangent to $M$. We have:

$$
\widehat{g}(X, X)= \begin{cases}\alpha-\alpha^{2}, & \text { if } \widetilde{T}^{2}=\widetilde{T} \\ -\alpha^{2}, & \text { if } \widetilde{T}^{2}=0 \\ -1-\alpha^{2}, & \text { if } \widetilde{T}^{2}=-I d\end{cases}
$$

The following proposition generalizes Corollary 4.1 of [1] (which concerns the case $\widetilde{T}^{2}=\widetilde{T}$ ). 
Proposition 2.10. The vector field $2 r X$ projects on a vector field on $M$ which is the gradient of $\alpha$ (with respect to the metric $g$ ).

Proof. Let $Z$ be a vector field on $M$. Denoting also by $Z$ its lift to $\widehat{M}$ and using Lemma 2.4, we have:

$$
D \alpha(Z)=2 T\left(\frac{1}{r} Z, \partial_{r}\right)=2 \widehat{g}\left(\frac{1}{r} Z, Y\right)=\frac{2}{r} \widehat{g}\left(Z, \alpha \partial_{r}+X\right)=g(Z, 2 r X) .
$$

Corollary 2.11. The gradient of $A$ is the vector field $2 r Y$. It satisfies

$$
\widehat{D}_{r Y} r Y= \begin{cases}r Y, & \text { if } \widetilde{T}^{2}=\widetilde{T}, \\ 0, & \text { if } \widetilde{T}^{2}=0, \\ -r \partial_{r}, & \text { if } \widetilde{T}^{2}=-I d\end{cases}
$$

and is therefore, in the first two cases, a pregeodesic vector field (i.e., up to reparameterization its integral curves are geodesics). The vector field $r X$ is pregeodesic for the metric $g$, more precisely we have

$$
D_{r X} r X= \begin{cases}(1-2 \alpha) r X, & \text { if } \widetilde{T}^{2}=\widetilde{T} \\ -2 \alpha r X, & \text { if } \widetilde{T}^{2}=0, \\ -2 \alpha r X, & \text { if } \widetilde{T}^{2}=-I d .\end{cases}
$$

Proof. We have $d A=2 r \alpha d r+r^{2} d \alpha$. Let $v=a \partial_{r}+h$ be a vector tangent to $\widehat{M}$ decomposed according to the splitting $T \widehat{M}=\mathbb{R} \partial_{r} \oplus T M$. We verify that $\widehat{g}(2 r Y,)=.d A$. Using Proposition 2.10, we have

$$
\begin{aligned}
d A(v) & =2 r \alpha a+r^{2} d \alpha(h) \\
& =2 r \alpha a+r^{2} g(2 r X, h) \\
& =2 r \widehat{g}\left(\alpha \partial_{r}+X, v\right) .
\end{aligned}
$$

The fact that the covariant derivative commutes with the musical isomorphisms $(\sharp$ and $b)$ implies $\widehat{g}(\widehat{D} .(2 r Y),$.$) is equal to the Hessian of A$. Hence $\widehat{g}\left(\widehat{D}_{r Y}(2 r Y),.\right)=2 T(r Y,$.$) and \widehat{D}_{r Y} r Y=r \widetilde{T}^{2}\left(\partial_{r}\right)$.

According to Fact $2.2, D_{r X} r X$ is the projection on $T M$ of $\widehat{D}_{r X} r X$. Using the fact that $r X=r Y-\alpha r \partial_{r}$ and Fact 2.2 again, it is straightforward to compute $\widehat{D}_{r X} r X$ and therefore $D_{r X} r X$. 
Corollary 2.12. The function $A$ is constant along the leaves of the foliation spanned by $\operatorname{ker} \widetilde{T}$.

Proof. It follows from the fact that for any $Z \in \operatorname{ker} \widetilde{T}$ we have $0=T\left(2 r \partial_{r}, Z\right)=$ $\widehat{g}(2 r Y, Z)=d A(Z)$.

\section{Description of the regular locus of $\alpha$}

Definition 3.1. Let $\alpha$ be a non-trivial solution of the Obata equation. We call regular locus of $\alpha$ the open dense set of $M$ defined by $M \backslash \alpha^{-1}(\sigma(T))$, where $\sigma(T)$ denotes the spectrum of $\widetilde{T}$ (hence in our case $\sigma(T) \subset\{0,1\}$ ).

We are able now to give a complete description of the regular locus of a solution $\alpha$. Everything starts from the following consequence of Section 2:

Corollary 3.2. Let $U$ be the open dense subset of $M$ defined by $U=\{m \in$ $\left.M \mid g_{m}(r X, r X) \neq 0\right\}$. The vector field $\bar{X}$ defined on $U$ by

$$
\bar{X}=-\frac{1}{\sqrt{|g(2 r X, 2 r X)|}} 2 r X
$$

is geodesic (i.e., it satisfies $D_{\bar{X}} \bar{X}=0$ ) and its local flow preserves the foliation of $U$ by level sets of $\alpha$. Moreover, if $\gamma$ is an integral curve of $\bar{X}$, there exists a constant $c$ such that we have

$$
\alpha(\gamma(s))= \begin{cases}\cos ^{2}(s+c), & \text { if } \widetilde{T}^{2}=\widetilde{T} \quad \text { and } \quad 0<\alpha<1, \\ \cosh ^{2}(s+c), & \text { if } \widetilde{T}^{2}=\widetilde{T} \text { and } \quad \alpha>1, \\ -\sinh ^{2}(s+c), & \text { if } \widetilde{T}^{2}=\widetilde{T} \text { and } \alpha<0, \\ e^{2 t+c}, & \text { if } \widetilde{T}^{2}=0 \text { and } \alpha>0, \\ -e^{-2 t+c}, & \text { if } \widetilde{T}^{2}=0 \text { and } \alpha<0, \\ \sinh (2 s+c), & \text { if } \widetilde{T}^{2}=-I d .\end{cases}
$$

Proof. The vector field $\bar{X}$ is pregeodesic and unitary, therefore it is geodesic. To see that it preserves the foliation of $U$ by level sets of $\alpha$, we compute $\bar{X} \cdot \alpha$. 
According to Proposition 2.10, we have $2 r X \cdot \alpha=g(2 r X, 2 r X)$ and therefore

$$
\begin{aligned}
\frac{1}{\sqrt{|g(2 r X, 2 r X)|} 2 r X \cdot \alpha}=-\frac{1}{\sqrt{|g(2 r X, 2 r X)|}} g(2 r X, 2 r X) \\
= \begin{cases} \pm 2 \sqrt{\left|\alpha-\alpha^{2}\right|}, & \text { if } \widetilde{T}^{2}=\widetilde{T}, \\
2|\alpha|, & \text { if } \widetilde{T}^{2}=0, \\
2 \sqrt{1+\alpha^{2}}, & \text { if } \widetilde{T}^{2}=-I d .\end{cases}
\end{aligned}
$$

The existence of such an equation implies that the local flow of $\bar{X}$ preserves the foliation. Solving these ordinary differential equations, we obtain the expression of $\alpha$ along an integral line of $\bar{X}$.

We recall that a pseudo-Riemannian manifold is said to be non-extendable if it is not isometric to a proper (i.e., non-closed) open subset of any other manifold. Clearly, a complete or compact pseudo-Riemannian manifold is non-extendable. Now, we can state the following theorem, it actually contains Theorems 1.1 and 1.3 given in the introduction.

Theorem 3.3. Let $(M, g)$ be a non-extendable pseudo-Riemannian manifold such that $(\widehat{M}, \widehat{g})$ has a parallel symmetric 2-tensor field $T$ such that $\widetilde{T}^{2}=\widetilde{T}, 0$ or $-\mathrm{Id}$. Let $\alpha$ be the function on $M$ associated to $T$ and $\sigma(T)$ be the spectrum of $\widetilde{T}$. Let $U$ be a connected component of $M \backslash \alpha^{-1}(\sigma(T))$.

If $(M, g)$ is complete or if all level sets of $\alpha$ on $U$ are compact, then there exist an open interval $I$, a pseudo-Riemannian manifold $(N, h)$ endowed with a parallel symmetric 2-tensor field $S$ (possibly trivial) such that $U=I \times N$ and

1. If $\widetilde{T}^{2}=0$, then $I=\mathbb{R}, \alpha(s, n)=e^{2 s}$ and

$$
\begin{aligned}
g & =-d s^{2}+\left(e^{2 s}(h-S)+S\right), \\
T_{\mid\{s\} \times N} & =e^{2 s} S .
\end{aligned}
$$

2. If $\widetilde{T}^{2}=-\mathrm{Id}$, then $I=\mathbb{R}, \alpha(s, n)=\sinh (2 s), U=M$ and

$$
\begin{aligned}
g & =-d s^{2}+(h-\sinh (2 s) S), \\
T_{\mid\{s\} \times N} & =S+\sinh (2 s) h .
\end{aligned}
$$

3. If $\widetilde{T}^{2}=\widetilde{T}$ and $0<\alpha<1$, then $\left.I=\right] 0, \pi / 2\left[, \alpha(s, n)=\cos ^{2}(s)\right.$ and

$$
\begin{aligned}
g & =d s^{2}+\left(\sin ^{2}(s)(h-S)+\cos ^{2}(s) S\right), \\
T_{\mid\{s\} \times N} & =\cos ^{2}(s) S .
\end{aligned}
$$


4. If $\widetilde{T}^{2}=\widetilde{T}$ and $\alpha>1$ (respectively $\alpha<0$ ), then $I=\mathbb{R}_{>0}$ (resp. $I=$ $\left.\mathbb{R}_{<0}\right), \alpha(s, n)=\cosh ^{2} s\left(\right.$ resp. $\left.\alpha(s, n)=-\sinh ^{2} s\right)$ and

$$
\begin{aligned}
g & =-d s^{2}+\left(\sinh ^{2}(s)(h-S)+\cosh ^{2}(s) S\right), \\
T_{\mid\{s\} \times N} & =\cosh ^{2}(s) S \\
\left(\text { resp. } T_{\mid\{s\} \times N}\right. & \left.=\sinh ^{2}(s)(h-S)\right) .
\end{aligned}
$$

Conversely, if $(N, h)$ is a pseudo-Riemannian manifold endowed with a parallel symmetric 2-tensor $S$ such that $\widetilde{S}^{2}=\widetilde{S}, 0$ or -Id then the pseudoRiemannian manifolds $(I \times N, g)$ given by the above formulas are well defined and the cone over any of them admits a non-trivial parallel symmetric 2-tensor field $T$.

Proof. We begin the proof by the following Lemma.

Lemma 3.4. Under the hypotheses of Theorem 3.3, and keeping the notation therein, there exist a manifold $N$ such that $U=\mathbb{R} \times N$. Moreover each submanifold $\{x\} \times N$ corresponds to a level of $\alpha_{\mid U}$ and the vector field $r X$ is everywhere tangent to the factor $\mathbb{R}$.

Proof of Lemma 3.4. Let $a$ and $b$ in $\mathbb{R}$ such that $a<b$ and $[a, b] \subset \alpha(U)$. Let $\varepsilon>0$ such that $[a-\varepsilon, b+\varepsilon] \subset \alpha(U)$. We denote by $K$ the subset of $U$ given by $K=\alpha^{-1}([a, b])$. We choose a bump function $l$ such that $l(K)=\{1\}$ and $l$ vanishes outside $\alpha^{-1}(] a-\varepsilon, b+\varepsilon[)$. As $g$ is geodesically complete or as $\alpha$ has compact levels, the vector field $l \bar{X}$ is complete.

Moreover, Corollary 3.2 says that the local flow of $\bar{X}$ preserves the foliation of $U$ by level set of $\alpha$. It entails that the flow of $l \bar{X}$ has the same property on $K$. We obtain that $\alpha^{-1}([a, b])$ is diffeomorphic to $J \times \alpha^{-1}(\{a\})$, where $J$ is a closed interval.

Thus $U$ is a fibre bundle over a one-dimensional manifold with fiber $N$ diffeomorphic to $\alpha^{-1}(\{a\})$. As $\alpha$ is constant along the fibers the base can not be compact, thus it is diffeomorphic to $\mathbb{R}$. The base being contractible, the fibre bundle is trivial, i.e., $U$ is diffeomorphic of $\mathbb{R} \times N$.

On the factor $\mathbb{R}$ given by Lemma 3.4, we choose a parameterization $t$ such that $\partial_{t}=r X$. The line is therefore identified with an open interval $J$. 
Lemma 3.5. We denote by $\pi$ the projection on $J$, by $S_{t}$ and $g_{t}$ the restrictions of $T$ and $g$ to $\{t\} \times N$, then

$$
\begin{aligned}
& g_{t}^{\prime}=-2 \bar{\alpha}(t) g_{t}+2 S_{t}, \\
& S_{t}^{\prime}=-2 \bar{\alpha}(t) S_{t}+2 S_{t}^{2},
\end{aligned}
$$

where $\bar{\alpha}$ is the function on $J$ such that $\bar{\alpha} \circ \pi=\alpha$ and $S^{2}$ is the 2-tensor field associated to the endomorphism $\widetilde{S}^{2}$. Moreover, denoting $D^{t}$ the Levi-Civita connexion of $g_{t}$, we have $D^{t} S_{t}=0$.

Proof of Lemma 3.5. If we denote by $\mathcal{L}_{r X}$ the Lie derivative according to $r X$, we have

$$
\mathcal{L}_{r X} g(u, v)=g\left(D_{u} r X, v\right)+g\left(D_{v} r X, v\right)=D^{2} \alpha(u, v)
$$

By Lemma 2.4 we thus have

$$
\mathcal{L}_{r X} g=-2 \alpha g+2 T
$$

which clearly implies (3.1).

On the other hand, by Lemma 2.4 we have

$$
D T(r X, u, v)=0
$$

for any $u, v$ perpendicular to $r X$. We deduce that if $u$ and $v$ are perpendicular to $r X$ then

$$
\begin{aligned}
\mathcal{L}_{r X} T(u, v) & =T\left(D_{u} r X, v\right)+T\left(D_{v} r X, u\right) \\
& =g\left(D_{u} r X, \widetilde{T}(v)\right)+g\left(D_{v} r X, \widetilde{T}(u)\right) \\
& =\frac{1}{2} D^{2} \alpha(u, \widetilde{T}(v))+\frac{1}{2} D^{2} \alpha(v, \widetilde{T}(u)) \\
& =-\alpha g(u, \widetilde{T}(v))+T(u, \widetilde{T}(v))-\alpha g(v, \widetilde{T}(u))+T(v, \widetilde{T}(u)) \\
& =-2 \alpha g(u, \widetilde{T}(v))+2 g\left(u, \widetilde{T}^{2}(v)\right) .
\end{aligned}
$$

This shows the second assertion.

Let $u, v$ and $w$ three vectors tangent to $\{t\} \times N$ at a point $(t, n)$. Using Lemma 2.4 we can write:

$$
0=D T(u, v, w)=D^{t} S_{t}(u, v, w)+T\left(I I_{t}(u, v) \bar{X}, w\right)+T\left(I I_{t}(u, w) \bar{X}, v\right)
$$


where $I I_{t}$ stands for the second fundamental form of $\{t\} \times N$. But for any $n \in \mathbb{N}$, we have $\widetilde{T}^{n}\left(\partial_{r}\right) \in \operatorname{Span}\left(\partial_{r}, r X\right)$ therefore $T(\bar{X}, u)=T(\bar{X}, v)=0$. Consequently, $D^{t} S_{t}=0$.

With our choice of parameter on $\mathbb{R}$ the metric reads $g=g(r X, r X) d t^{2}+$ $g_{t}$. This is not the desired shape. Thus we need to reparameterize $\mathbb{R}$, we choose a parameter $s$ such that $\partial_{s}=\bar{X}$. The equations obtained at Lemma 3.5 turn into:

$$
\begin{aligned}
g_{s}^{\prime} & =\frac{-1}{\sqrt{|g(r X, r X)|}}\left(-2 \bar{\alpha}(s) g_{s}+2 S_{s}\right), \\
S_{s}^{\prime} & =\frac{-1}{\sqrt{|g(r X, r X)|}}\left(-2 \bar{\alpha}(s) S_{s}+2 S_{s}^{2}\right) .
\end{aligned}
$$

If $\widetilde{T}^{2}=0$, according to Corollary 3.2 , we obtain the equations

$$
\begin{aligned}
& g_{s}^{\prime}=\epsilon 2 g_{s}-2 e^{-\epsilon s} S_{s}, \\
& S_{s}^{\prime}=\epsilon 2 S_{s},
\end{aligned}
$$

where $\epsilon$ is the sign of $\alpha$.

If $\widetilde{T}^{2}=-\mathrm{Id}$, according to Corollary 3.2 , we obtain the equations

$$
\begin{aligned}
& g_{s}^{\prime}=2 \tanh (2 s) g_{s}-\frac{2}{\cosh (2 s)} S_{s} \\
& S_{s}^{\prime}=2 \tanh (2 s) S_{s}+\frac{2}{\cosh (2 s)} g_{s} .
\end{aligned}
$$

If $\widetilde{T}^{2}=\widetilde{T}$ and $0<\alpha<1$, according to Corollary 3.2 , we obtain the equations

$$
\begin{aligned}
g_{s}^{\prime} & =\frac{2}{\tan (s)} g_{s}-\frac{2}{\cos (s) \sin (s)} S_{s}, \\
S_{s}^{\prime} & =-2 \tan (s) S_{s} .
\end{aligned}
$$

If $\widetilde{T}^{2}=\widetilde{T}$ and $\alpha<0$, according to Corollary 3.2 , we obtain the equations

$$
\begin{aligned}
g_{s}^{\prime} & =\frac{2}{\tanh (s)} g_{s}-\frac{2}{\cosh (s) \sinh (s)} S_{s}, \\
S_{s}^{\prime} & =2 \tanh (s) S_{s} .
\end{aligned}
$$

The case $\widetilde{T}^{2}=\widetilde{T}$ and $\alpha>1$ is similar to the later.

There exists a unique solution to each of these systems of differential equations with given initial data. Now there is no difficulty to check that 
these solutions are the one given in the statement. If the interval $I$ is smaller than the one given in the statement then $(M, g)$ is clearly extendable. Thus, we proved the first half of the theorem. Let us remark that the endomorphisms $\widetilde{S}_{s}$ do not depend on $s$.

For the converse, the first thing to check is that the metrics $g_{s}$ given in the statement are never degenerate. As it is a pointwise property, it is linear algebra. We denote by $I_{k}$ the identity matrix of order $k$, and by $N$ the matrix $\left(\begin{array}{ll}0 & 1 \\ 0 & 0\end{array}\right)$. Let $\widetilde{S}$ and $\widetilde{g}_{s}$ such that $S=h(\widetilde{S} .,$.$) and g_{s}=h\left(\widetilde{g}_{s} .,.\right)$. It is easy to find a frame of $T_{p} N$ such that the matrix of $\widetilde{S}$ is given by

$$
\begin{aligned}
& \left(\begin{array}{cc}
I_{k} & 0 \\
0 & 0
\end{array}\right), \quad \text { when } \widetilde{S}^{2}=\widetilde{S}, \\
& \left(\begin{array}{llll}
N & & & \\
& \ddots & & \\
& & N & \\
& & & 0
\end{array}\right), \quad \text { when } \widetilde{S}^{2}=0 \text {, } \\
& \left(\begin{array}{cc}
0 & -I_{\frac{n}{2}} \\
I_{\frac{n}{2}} & 0
\end{array}\right), \quad \text { when } \widetilde{S}^{2}=- \text { Id } \text {. }
\end{aligned}
$$

The matrix in those frames of $\widetilde{g}_{s}$ are now easy to write down. They are clearly non-degenerate.

If there exists a parallel tensor $T$ on $\widehat{M}$ inducing $S$ on $N$, then there exists a vector field $Y$ such that the endomorphism $\widetilde{T}$ is given by :

$$
\left\{\begin{array}{l}
\widetilde{T}\left(\partial_{r}\right)=Y, \\
\left.\widetilde{T}(Y)=0,-\partial_{r} \text { or } Y \quad \text { (according to the relation between } \widetilde{S} \text { and } \widetilde{S}^{2}\right), \\
\widetilde{T}(Z)=\widetilde{S}(Z), \quad \text { for any vector } Z \text { tangent to }\{s\} \times N .
\end{array}\right.
$$

As we already know the function $\alpha$, it is not difficult to give $Y$. It has to be:

$$
Y=\alpha(s) \partial_{r}-\frac{1}{r} \sqrt{\beta(s)} \partial_{s}
$$

where

$$
\beta= \begin{cases}\left|\alpha-\alpha^{2}\right|, & \text { if } \widetilde{T}^{2}=\widetilde{T} \\ \alpha^{2}, & \text { if } \widetilde{T}^{2}=0 \\ 1+\alpha^{2}, & \text { if } \widetilde{T}^{2}=- \text { Id }\end{cases}
$$

We have only to check that the tensor field $T$ on $\widehat{M}$ given in the statement is parallel. The main difficulty is solved by the following Lemma. 
Lemma 3.6. Let $\left(g_{t}, S_{t}\right)$ be path of metrics and symmetric 2-tensors on a manifold $N$ satisfying (3.1) and (3.2) and such that $D^{t_{0}} S_{t_{0}}=0$ and $\widetilde{S}_{t_{0}}^{2}=0$, -Id or $\widetilde{S}_{t_{0}}$. Then for any $t$ we have $D^{t} S_{t}=0$.

Proof of Lemma 3.6. The first point is that $D^{t_{0}} S_{t}$ and $D^{t_{0}} g_{t}$ are solutions of a differential equation. Indeed we have:

$$
\begin{aligned}
\left(D^{t_{0}} g_{t}\right)^{\prime} & =D^{t_{0}}\left(g_{t}^{\prime}\right)=-2 \alpha(t) D^{t_{0}}\left(g_{t}\right)+2 D^{t_{0}}\left(S_{t}\right) \\
\left(D^{t_{0}} S_{t}\right)^{\prime} & =D^{t_{0}}\left(S_{t}^{\prime}\right)=-2 \alpha(t) D^{t_{0}}\left(S_{t}\right)+2 D^{t_{0}}\left(S_{t}^{2}\right)
\end{aligned}
$$

As $\widetilde{S}_{t}^{2}=0,-$ Id or $\widetilde{S}_{t}$, this equation is in fact linear. But for $t=t_{0}$ we have $D^{t_{0}} g_{t_{0}}=0$ and $D^{t_{0}} S_{t_{0}}=0$; therefore for any $t$ we have $D^{t_{0}} g_{t}=0$ and $D^{t_{0}} S_{t}=0$. It means that $D^{t_{0}}=D^{t}$ and $D^{t} S_{t}=0$.

We leave to the reader the last verifications.

As we said in the introduction, the case $S=0$ of part 3.3 of Theorem 3.3 can be find in [1]. The parts 3.3 and 3.3 of Theorem 3.3 have also already appeared in [1] under a slightly different form. To obtain the former version, we just have to apply the De Rham-Wu Theorem, locally or globally, to the triplet $(N, h, S)$. However, in order to write down the global version, we need an assumption of completeness on $N$ (see [9]), except in the Riemannian or in the Lorentzian case it is not enough to suppose the manifold compact. It is undoubtedly a very interesting question to know if there exist decomposable compact pseudo-Riemannian manifolds whose universal cover is not a product.

Corollary 3.7. Let $(M, g)$ be a pseudo-Riemannian manifold such that its cone $(\widehat{M}, \widehat{g})$ has a parallel symmetric 2 -tensor field $T$ such that $\widetilde{T}^{2}=\widetilde{T}$ (i.e., $(M, g)$ has a decomposable cone). Let $U$ be a connected component of $M \backslash \alpha^{-1}(\{0,1\})$ and $\widetilde{U}$ denote its universal cover. If the foliation defined by the kernel of the restriction of $T$ to $M$ is geodesically complete (for example if $g$ is geodesically complete or if $\alpha$ has compact levels and $\operatorname{ker} \widetilde{T}$ is spacelike) then there exists two pseudo-Riemannian manifolds $\left(N_{1}, h_{1}\right)$ and $\left(N_{2}, h_{2}\right)$ such that

$$
\begin{aligned}
\tilde{U} & =I \times N_{1} \times N_{2}, \\
\text { and } \quad g & =d s^{2}+\cos ^{2}(s) h_{1}+\sin ^{2}(s) h_{2}, \\
\text { or } \quad g & =-d s^{2}+\cosh ^{2}(s) h_{1}+\sinh ^{2}(s) h_{2} .
\end{aligned}
$$




\section{Decomposable cones}

In this section, we suppose that $\widehat{M}$ is decomposable or equivalently that $\widetilde{T}^{2}=\widetilde{T}$. We will also assume that $(M, g)$ is complete or that $\alpha$ is proper. The assumption of properness is stronger than the assumption that $\alpha$ has compact levels. It is mainly a way to say that no connected component of the levels $\alpha^{-1}(0)$ or $\alpha^{-1}(1)$ is missing. It follows from Theorem 3.3 that the image of $\alpha$ is a union of sets chosen among the following ones: $]-\infty, 0[,\{0\}$, ] $0,1[,\{1\}] 1,,+\infty[$.

Definition 4.1. We denote by $\mathcal{F}$ the foliation of $\widehat{M}$ spanned by $\operatorname{ker} \widetilde{T}$ and by $\mathcal{G}$ the foliation defined by $\operatorname{Im} \widetilde{T}$.

A parallel distribution being integrable these foliations do exist. Moreover, their roles are symmetric as it is always possible to replace $\widetilde{T}$ by $\operatorname{Id}-\widetilde{T}$. These foliations will play an important role in what follows.

To begin with, we suppose that the function $\alpha$ is bounded and that $(M, g)$ is complete. Actually, Theorem 4.2 is almost proven in [1] by following Gallot's proof from [5]. The missing point (the fact that the metric is Riemannian) is in [7]. But for the convenience of the reader, we recall its proof (except for the Lemmas 4.3 and 4.4 that are stated without proof). We modified the presentation of some arguments in order to make clear how it is possible to adapt it to the case where $\alpha$ is supposed proper.

Theorem 4.2. Let $(M, g)$ be a complete pseudo-Riemannian manifold with decomposable cone. We have $0 \leq \alpha(m) \leq 1$ for all $m \in M$ if and only if $(M, g)$ is finitely covered by a Riemannian round sphere.

Proof. We suppose $(M, g)$ complete. Let $Y=\widetilde{T}\left(\partial_{r}\right), \Gamma$ be the geodesic of $(\widehat{M}, \widehat{g})$ starting from the point $\left(r_{0}, m_{0}\right)$ in the direction $-r Y\left(r_{0}, m_{0}\right)$ and $\gamma$ the geodesic of $(M, g)$ starting from $m_{0}$ in the direction $-r X\left(m_{0}\right)$. Let us remark that $\Gamma^{\prime}(t)$ is never lightlike and that, by Corollary $2.11, Y(\Gamma(t))$ is always proportional to $\Gamma^{\prime}(t)$.

As in [1] and [5] we prove that $\Gamma$ contains a point where $Y$ vanishes.

Lemma 4.3 (see [1], section 5). We denote by $\alpha_{0}$ the number $\alpha\left(m_{0}\right)$. We have $\gamma(t)=(r(t), \gamma(f(t)))$, with

$$
r(t)=\sqrt{\left(\alpha_{0} t+r_{0}\right)^{2}+\left(\alpha_{0}-\alpha_{0}^{2}\right) r_{0}^{2} t^{2}}
$$




$$
\begin{aligned}
& f(t)=\frac{1}{\sqrt{\alpha_{0}-\alpha_{0}^{2}}} \arctan \left(\frac{\sqrt{\left(\alpha_{0}-\alpha_{0}^{2}\right) r_{0} t}}{\alpha_{0} t+r_{0}}\right), \quad \text { if } \quad \alpha_{0}-\alpha_{0}^{2}>0, \\
& f(t)=\frac{1}{\sqrt{\alpha_{0}^{2}-\alpha_{0}}} \operatorname{argtanh}\left(\frac{\sqrt{\alpha_{0}^{2}-\alpha_{0} r_{0} t}}{\alpha_{0} t+r_{0}}\right), \quad \text { if } \quad \alpha_{0}-\alpha_{0}^{2}<0 .
\end{aligned}
$$

The geodesic $\Gamma$ is thus defined on $[0,1]$. We deduce from Corollary 2.11 that

$$
A \circ \Gamma^{\prime}(t)=-2 r_{0} \sqrt{\alpha_{0}} \sqrt{A \circ \Gamma(t)} .
$$

Therefore, $A \circ \Gamma(t)=\left(-r_{0}^{2} \alpha_{0} t^{2}+r_{0}^{2} \alpha_{0}\right)$ and $A \circ \Gamma(1)=0$. It implies that $\widehat{g}(Y(\Gamma(1)), Y(\Gamma(1)))=0$. As $Y(\Gamma(1))$ can not be lightlike, it has to be zero.

The point $\Gamma(1)$ is a minimum for $A$ therefore its Hessian is positive, but (cf. Proposition 2.3) $\widehat{D} \widehat{D} A=2 T$ and $\widetilde{T}$ is a projector. This means that the restriction of $\widehat{g}$ to $\operatorname{Im} \widetilde{T}$ is Riemannian.

But, we can replace $\widetilde{T}$ by $\mathrm{Id}-\widetilde{T}$ and repeat this proof. We obtain that the restriction of $\widehat{g}$ to $\operatorname{ker} \widetilde{T}$ is also Riemannian.

It is well known (and it follows from Fact 2.2) that the curvature of $\widehat{g}$ is given by

$$
\widehat{R}(X, Y) Z=R(X, Y) Z-g(Y, Z) X+g(X, Z) Y,
$$

where $R$ and $\widehat{R}$ are the curvatures of $g$ and $\widehat{g}$. It implies that $(\widehat{M}, \widehat{g})$ is flat if and only if $(M, g)$ has constant curvature equal to 1 .

To prove that $\widehat{g}$ is flat, we use the following Lemma from [5] and [1].

Lemma 4.4 ([5] Lemma 3.2 or [1] Lemma 6.3). If $Y(r, m)=0$ then the leaf of $\mathcal{G}$ (the foliation spanned by $\operatorname{Im} \widetilde{T}$ ) containing $(r, m)$ is flat.

We have proven that $\mathcal{G}$ is flat. In order to prove that $(\widehat{M}, \widehat{g})$ is flat, we have to show that $\mathcal{F}$ is also flat. It is done by repeating the proof with the tensor field $\widehat{g}-T$ in place of $T$.

As we said at the beginning of the section, we are also interested in replacing the assumption of geodesic completeness by the assumption that $\alpha$ is proper. To adapt the proof above to this case, we just have to prove the following proposition:

Proposition 4.5. Let $m \in M$ such that $\alpha(m)<1$ and $\alpha(m) \neq 0$ and let $G_{(r, m)}$ be the leaf of $\mathcal{G}$ containing the point $(r, m)$. If $\alpha$ is proper then there exists a point $p$ in $G_{(r, m)}$ such that $Y(p)=0$. 
Proof. The proof starts the same way. We are looking for a critical point of the restriction of $A$ to $G_{(r, m)}$. Classically, we follow (backward) the integral curves of the gradient. We note that the gradient of the restriction of $A$ is also $2 r Y$ because $Y$ is tangent to $\mathcal{G}$. Let $(r, \gamma):] a, b[\rightarrow G(r, m)$ be the maximal integral curve of $-r Y$ such that $(r(0), \gamma(0))=(r, m)$.

Lemma 4.6. The image of the restriction of $\gamma$ to $[0, b[$ lies in a compact set of $\widehat{M}$.

Proof. The value of $\alpha$ is bounded along this curve, as $\alpha$ is proper, we just have to show that $r(] a, 0])$ is contained in a compact subset of $] 0,+\infty[$.

We suppose $\alpha(m)>0$ (respectively $\alpha(m)<0$ ). First, we remark that $-r Y \cdot r=-r \alpha \leq 0$ (resp. $\geq 0$ ). This implies that

$$
\forall t \in[0, b[, r(t) \leq r(0)=r(\text { resp. } r(t) \geq r(0)) .
$$

We thus have a upper bound (resp. lower bound) on $r(t)$.

If we apply Corollary 2.12 to the parallel tensor field $\widehat{g}-T$, we obtain that the function $r^{2}(1-\alpha)$ is constant along the leaves of $\mathcal{G}$.

Hence, if $\left(r^{\prime}, m^{\prime}\right)$ is a point of $G_{(r, m)}$ we have $r^{2}\left(1-\alpha\left(m^{\prime}\right)\right)=r^{2}(1-$ $\alpha(m)) \neq 0$. Moreover $1-\alpha\left(m^{\prime}\right) \leq 1 \quad\left(\right.$ resp. $1-\alpha\left(m^{\prime}\right) \geq 1$ ) therefore $r^{\prime} \geq r_{0} \sqrt{1-\alpha(m)}\left(\operatorname{resp} r^{\prime} \leq r_{0} \sqrt{1-\alpha(m)}\right)$. As for all $\left.t \in\right] a, b[$, we have $(r(t), \gamma(t)) \in G_{(r, m)}$, this gives a lower bound (resp. a upper bound) for $r(t)$.

It follows from Lemma 4.6 that there exists a sequence $\left(t_{n}\right)_{n \in \mathbb{N}}$ of points of $\left[0, b\left[\right.\right.$ converging to $b$ and such that the sequence $\left(\gamma\left(t_{n}\right)\right)_{n \in \mathbb{N}}$ converges in $\widehat{M}$ to a point $\left(r_{\infty}, m_{\infty}\right)$. Let $U$ be a foliated neighborhood for $\mathcal{G}$ of $\left(r_{\infty}, m_{\infty}\right)$. There are two possibilities: either $\left(r_{\infty}, m_{\infty}\right)$ belongs to $G_{(r, m)}$ or the points $\gamma\left(t_{n}\right)$ belong to an infinite number of connected components of $U \cap G_{(r, m)}$ (called plaques). The last case implies that the leaf $G_{(r, m)}$ accumulates around $\left(r_{\infty}, m_{\infty}\right)$. As the vector $\partial_{r}$ is never tangent to $G_{(r, m)}$ this is incompatible with the following straightforward consequence of Corollary 2.12.

Fact 4.7. Let $m \in M$, if the set $\mathbb{R}_{>0} \times\{m\} \cap G_{(r, m)}$ contains more than one point then $\mathbb{R}_{>0} \times\{m\} \subset G_{(r, m)}$.

Hence $\left(r_{\infty}, m_{\infty}\right) \in G_{(r, m)}$ and is therefore a critical point of the restriction of $A$ to $G_{(r, m)}$. It means that the gradient vanishes at this point, i.e., that $Y\left(r_{\infty}, m_{\infty}\right)=0$. 
Replacing $T$ by $\widehat{g}-T$, we get:

Corollary 4.8. Let $m \in M$ such that $\alpha(m)>0$ and $\alpha(m) \neq 1$ and let $F_{(r, m)}$ be the leaf of $\mathcal{F}$ containing the point $(r, m)$. If $\alpha$ is proper then there exists a point $p$ in $F_{(r, m)}$ such that $Y(p)=\partial_{r}$.

We can therefore replace the hypothesis " $g$ is complete" by " $\alpha$ is proper" and repeat the proof of Theorem 4.2. However, if $\alpha$ is bounded and proper then $M$ is actually compact. Therefore, we can use Proposition 2.7 to improve the statement. We get the following statement which is actually the main result of [7]:

Corollary 4.9 (see [7] Theorem 1). If $(M, g)$ is closed (compact without boundary) and if its cone admits a non-trivial parallel symmetric 2-tensor field then $(M, g)$ is finitely covered by a Riemannian round sphere.

Proof. Proposition 2.7 says that if $M$ is compact then $(\widehat{M}, \widehat{g})$ is decomposable. The manifold $M$ being closed, there exists $\left(m_{+}, m_{-}\right) \in M^{2}$ such that $\alpha\left(m_{+}\right)=\max _{m \in M} \alpha(m)$ and $\alpha\left(m_{-}\right)=\min _{m \in M} \alpha(m)$, therefore $d \alpha\left(m_{ \pm}\right)=$ 0 . According to Corollary 2.8, the only critical values of $\alpha$ are 0 and 1 therefore $\alpha\left(m_{-}\right)=0$ and $\alpha\left(m_{+}\right)=1$. Then Proposition 4.5 enable us to adapt the proof of Theorem 4.2.

There is a shorter way to prove Corollary 4.9. It consists in proving first that $(M, g)$ is Riemannian and therefore complete (or apply the Gallot-Tano Theorem). It is what is done in [7]. Anyway, our purpose was rather to use Proposition 4.5 than to give a proof of Corollary 4.9 .

The following result extends Theorem 4.2, together they say that, hitherto, there are only trivial examples.

Theorem 4.10. Let $(M, g)$ be a connected pseudo-Riemannian manifold with decomposable cone and $\alpha$ be the associated solution of the Obata equation. If $(M, g)$ is complete or if $\alpha$ is proper and if there exists $m \in M$ such that $0<\alpha(m)<1$ then $(M, g)$ has constant curvature equal to 1 .

Proof. We assume $(M, g)$ complete but using Proposition 4.5, it is easy to adapt the proof to the case where $\alpha$ is proper.

The first step consists in repeating the proof of Theorem 4.2. Doing so we obtain that the metric $\widehat{g}$ is flat on $\mathbb{R}_{>0} \times \alpha^{-1}(] 0,1[$ ) (it was also proven in $[1])$.

Let $p \in \widehat{M}$ be a point such that $Y(p)=0$ (resp. $Y(p)=\partial_{r}$ ). We are going to see that the curvature vanishes at $p$. Corollary 2.9 tells us that $p$ belongs 
to the closure of $\mathbb{R}_{>0} \times \alpha^{-1}(] 0,1[$ ) (and the curvature of $\widehat{g}$ therefore vanishes at $p$ ) unless $p$ is a local maximum (resp. minimum). But in that case the restriction of $\widehat{g}$ to $\operatorname{Im} \widetilde{T}$ (resp. $\operatorname{ker} \widetilde{T}$ ) is negative Riemannian. Hence $Y(p)$ (resp. $\left.Y(p)-\partial_{r}\right)$ is never lightlike. Therefore if $\alpha(m)=0(\operatorname{resp} \alpha(m)=1)$ then $Y(r, m)=0\left(\operatorname{resp} . Y(p)=\partial_{r}\right)$. It means that for all $m \in M$ we have $\alpha(m)<0$ (resp. $\alpha(m)>1$ ) and this contradicts our hypotheses.

We suppose there exists a point $m \in M$ such that $\alpha(m)<0$ and we choose $r>0$, then according to Lemma 4.3 the geodesic starting from $(r, m)$ with initial speed $-r Y$ (which is contained in a leaf of $\mathcal{G}$ ) is defined on $[0,1]$. Hence, reproducing the proof above, it contains a point $p$ such that $Y(p)=0$, therefore it follows from Lemma 4.4 that the curvature of $\widehat{g}$ vanishes along the leaf of $\mathcal{G}$ containing $(r, m)$. But, as we just saw, the curvature of $\widehat{g}$ vanishes at $p$. The two points $p$ and $(r, m)$ lie in the same leaf of $\mathcal{G}$. As the distribution $\operatorname{ker} \widetilde{T}$ is parallel and is perpendicular to $\mathcal{G}$, the curvature of the restriction of $\widehat{g}$ to $\operatorname{ker} \widetilde{T}$ is the same at $p$ and at $(r, m)$. It proves that $\widehat{g}$ is flat at $(r, m)$, therefore that $\widehat{g}$ is flat on $\left.\left.\alpha^{-1}(]-\infty, 0\right]\right)$.

To study the set $\alpha^{-1}([1,+\infty[)$, we consider the endomorphism $\operatorname{Id}-\widetilde{T}$. The function associated to it is $1-\alpha$, hence this case is similar to the former. We proved that $\widehat{g}$ is flat therefore that $g$ has constant curvature equal to 1 .

The last case needs more work, in particular we need a better understanding of the set $\alpha^{-1}(\{0,1\})$. It is also more interesting, as it provides examples with non-constant curvature.

Theorem 4.11. Let $(M, g)$ be a connected pseudo-Riemannian manifold with decomposable cone and $\alpha$ be the associated solution of the Obata equation. We suppose $g$ is complete or $\alpha$ is proper. If for all $m \in M, \alpha(m) \leq 0$ or $\alpha(m) \geq 1$ then there exists a pseudo-Riemannian manifold $\left(F, g_{F}\right)$ such that:

- either up to a 2-cover, $M=\mathbb{R} \times F$ and $g=-d s^{2}+\cosh ^{2}(s) g_{F}$;

- or $\widetilde{M}$, the universal cover of $M$, is a warped product of the negative definite $n$-dimensional hyperbolic space and $\left(F, g_{F}\right)$.

More precisely $\widetilde{M}$ is diffeomorphic to $\mathbb{R}^{n} \times F$ and using polar coordinates on $\mathbb{R}^{n}$ the metric $g$ is given by $g=-d s^{2}-\sinh ^{2}(s) g_{S}+$ $\cosh ^{2}(s) g_{F}$, where $g_{S}$ is the standard metric of the $(n-1)$-sphere.

Moreover, there exist a group $\Lambda$ acting freely and properly discontinuously on $F$, a morphism $\rho$ from $\Lambda$ to the group of isometries of $\mathbb{H}_{-}^{n}$ fixing a given point such that $M=\left(\mathbb{H}_{-}^{n} \times F\right) /(\rho(\Lambda), \Lambda)$. 
Conversely, the manifolds above have a decomposable cone. Furthermore, they are complete if and only if $\left(F, g_{F}\right)$ is complete and they admit a proper solution to the Obata equation if and only if $F / \Lambda$ or $F$ is compact.

Proof. If we replace $\widetilde{T}$ by $\mathrm{Id}-\widetilde{T}$, we permute the cases $\alpha(m) \geq 1$ and $\alpha(m) \leq 0$. Thus, without loss of generality, we will now suppose that for all $m \in M, \alpha(m) \leq 0$. We start the proof by the following lemma.

Lemma 4.12. Let $m$ be a point of $M$ and $\gamma$ be the geodesic such that $\gamma(0)=m$ and $\gamma^{\prime}(0)=\bar{X}(m)$ then there exists $t \in \mathbb{R}$ such that $\alpha(\gamma(t))=0$. The set $\alpha^{-1}(0)$ is a submanifold of $M$ and its dimension is $\operatorname{dim}(\operatorname{ker} T)-1$. The restriction of $\widehat{g}$ to $\operatorname{Im} \widetilde{T}$ is negative definite.

Proof. The first point is given by Proposition 4.5 when $\alpha$ is proper and by Lemma 4.3 and the discussion that follows when $g$ is complete.

As 0 is the maximum of $\alpha$, any element of $\alpha^{-1}(0)$ is a critical point. By the argument used in the proof of Theorem 4.2, it entails that the restriction of $\widehat{g}$ to $\operatorname{Im} \widetilde{T}$ is negative definite.

Furthermore, it follows from Corollary 2.12 that if $\alpha(m)=0$ then $A$ vanishes on any leaf of $\mathcal{F}$ (the foliation spanned by ker $\widetilde{T}$ ) containing a point $(r, m)$. According to Proposition 2.3, the Hessian of $A$ is twice the restriction of $\widehat{g}$ to $\operatorname{Im} \widetilde{T}$. It means that the singular points of the restriction of $A$ to any leaf of $\mathcal{G}$ (the foliation spanned by $\operatorname{Im} \widetilde{T}$ ) are non-degenerate and therefore isolated. Hence, $A^{-1}(0)$ is a union of isolated leaves of $\mathcal{F}$. Moreover, the vector field $\partial_{r}$ is everywhere tangent to these leaves. The set $\alpha^{-1}(0)$ being the projection of $A^{-1}(0)$, it is therefore a $(\operatorname{dim}(\operatorname{ker} \widetilde{T})-1)$-dimensional smooth submanifold.

Lemma 4.13. The projection of the distribution $\operatorname{Im} \widetilde{T}$ to $M$ is a smooth, integrable, totally geodesic and non-degenerate distribution. We denote it by $V$ and by $\mathcal{G}^{\prime}$ the foliation it defines. If $n=\operatorname{dim}(\operatorname{Im} \widetilde{T})>1$, then any leaf of $\mathcal{G}^{\prime}$ is isometric to the negative definite hyperbolic space $\mathbb{H}_{-}^{n}$.

Proof. As $\partial_{r}$ is geodesic and $\operatorname{Im} \widetilde{T}$ is parallel, we know that the projection of $\operatorname{Im} \widetilde{T}(r, m)$ on $T_{m} M$ does not depend on $r$. Moreover, $\operatorname{Im} \widetilde{T}$ never contains $\partial_{r}$; therefore the distribution $V$ is smooth and integrable. The restriction of $\widehat{g}$ to $\operatorname{Im} \widetilde{T}$ is negative definite, therefore the restriction of $g$ to $V$ is also negative definite.

Let $Z, Z^{\prime}$ be two vector fields tangent to $V$. Their lift to $\widehat{M}$, still denoted by $Z, Z^{\prime}$ lie in $\operatorname{Im} \widetilde{T} \oplus \mathbb{R} \partial_{r}$. This last distribution is clearly totally geodesic. 
Moreover, from Fact 2.2, we know that $\widehat{D}_{Z} Z^{\prime}=D_{Z} Z^{\prime}-r g\left(Z, Z^{\prime}\right) \partial_{r}$. It means that $D_{Z} Z^{\prime}$ is tangent to $V$ and therefore that $V$ is totally geodesic.

Let $u$ and $v$ be two vectors of $T_{m} M$, from Lemma 2.4 we have

$$
2 T(u, v)=\widehat{D} \widehat{D} A(u, v)=r^{2}(D D \alpha(u, v)+2 g(u, v) \alpha(m)) .
$$

If $m$ is a critical point of $\alpha$, we have $\alpha(m)=0$ and $\operatorname{Im} \widetilde{T}$ perpendicular to $\partial_{r}$. Hence, the Hessian of $\alpha$ at $m$ is given by the restriction of $g$ to $V_{m}$. Therefore, the restriction of $\alpha$ to any leaf $G^{\prime}$ of $\mathcal{G}^{\prime}$ is a Morse function and the critical points of $\alpha_{\mid G^{\prime}}$ are isolated. In particular, when $n>1$, the set of regular points of the restriction of $\alpha$ to $G^{\prime}$ is connected.

Moreover, as the vector field $2 r X$ is tangent to $\mathcal{G}^{\prime}$, it is also the gradient of $\alpha_{\mid G^{\prime}}$. Hence (using geodesic completeness or the properness of $\alpha$ ) there exists a critical point of $\alpha$ in the closure of $G^{\prime}$. This critical point belongs to $\alpha^{-1}(0)$, which is transverse to $\mathcal{G}^{\prime}$, therefore the point lies on $G^{\prime}$.

We use now the vector field $\bar{X}=-r X / \sqrt{-g(r X, r X)}$, which is defined on $M \backslash \alpha^{-1}(0)$. Let $c_{1}<c_{2}<0$ and $\varepsilon>0$ such that $c_{2}+\varepsilon<0$. Let $l$ be a function vanishing outside $\alpha^{-1}(] c_{1}-\varepsilon, c_{2}+\varepsilon[)$ and being constant equal to one on $\alpha^{-1}(] c_{1}, c_{2}[)$. The vector field $l \bar{X}$ is complete in both cases. According to Corollary 3.2, its flow restricted to $\alpha^{-1}(] c_{1}, c_{2}[)$ sends level sets of $\alpha$ on level sets of $\alpha$. It follows that any two regular level sets of $\alpha_{\mid G^{\prime}}$ are diffeomorphic.

If $\left(\alpha_{\mid G^{\prime}}\right)^{-1}\left(c_{1}\right)$ is not connected, we can saturate its connected components by the gradient lines in order to obtain a partition of the set of regular points of $G^{\prime}$. This set being connected, we have a contradiction, therefore the level sets of $\alpha_{\mid G^{\prime}}$ are connected.

Hence, as $\alpha_{\mid G^{\prime}}$ is a Morse function, any level set of the restriction of $\alpha$ to a leaf of $\mathcal{G}^{\prime}$ is diffeomorphic to the sphere $S^{n-1}$. Consequently, the leaves of $\mathcal{G}^{\prime}$ are diffeomorphic to $\mathbb{R}^{n}$.

We have shown that the function $\alpha$ vanishes on any leaf of $\mathcal{G}^{\prime}$ therefore the function $A$ vanishes on any leaf of $\mathcal{G}$. It follows from Lemma 4.4 that the curvature of $\widehat{g}$ vanishes along $\operatorname{Im} \widetilde{T}$, and, by Fact 2.2 , that the curvature of $g$ is constant and equal to one on $V$ (this point is proven in [1]).

Thus, we have shown that any leaf $G^{\prime}$ of $\mathcal{G}^{\prime}$ is diffeomorphic to $\mathbb{R}^{n}$ and that $g_{\mid G^{\prime}}$ is negative definite and has constant curvature equal to 1 . As, moreover, the flow of $\bar{X}$ is future complete (even if we suppose $\alpha$ proper), it means that any leaf of $\mathcal{G}^{\prime}$ is isometric to $\mathbb{H}_{-}^{n}$.

We denote by $W$ the orthogonal complement of $V$ in $T M$. This distribution is integrable, its leaves being the intersection of the level sets of $\alpha$ 
(which are all smooth) with the projection on $M$ of the leaves of $\mathcal{F}$. We denote by $\mathcal{F}^{\prime}$ the foliation spanned by $W$. In particular, $\alpha^{-1}(0)$ is a union of leaves of $\mathcal{F}^{\prime}$.

We assume now that $n=\operatorname{dim}(\operatorname{Im} \widetilde{T})=1$. Taking eventually a 2-cover, the direction field given by the projection of $\operatorname{Im} \widetilde{T}$ is well defined and oriented. Moreover, the vector field $2 r X$ belongs to this field. It means that it is possible to extend to $M$ the vector field $\bar{X}=r X / \sqrt{-g(r X, r X)}$. The function $\alpha$ being unbounded, Corollary 3.2 implies that this vector field has to be complete. Always by Corollary 3.2, its flow sends levels of $\alpha$ on levels of $\alpha$. Moreover, the leaves of $\mathcal{G}^{\prime}$ cannot be compact, therefore there exists a manifold $F$ such that $M$ is diffeomorphic to $\mathbb{R} \times F$ (see Lemma 3.4). Eventually, Corollary 3.7 (see also Theorem 7.1 from [1]) shows that $g$ is given on $\mathbb{R}^{*} \times F$ by $-d s^{2}+\cosh ^{2}(s) g_{F}$, where $g_{F}$ is a metric on $F$. The first assertion of Theorem 4.11 follows then by continuity.

We assume now that $n=\operatorname{dim}(\operatorname{Im} \widetilde{T})>1$.

Fact 4.14. The submanifold $\alpha^{-1}(0)$ is connected. The universal cover of $M$ is diffeomorphic to $\mathbb{R}^{n} \times F$, where $F$ is the universal cover of $\alpha^{-1}(0)$.

Proof. The foliation $\mathcal{G}^{\prime}$ is totally geodesic, complete (it is isometric to $\mathbb{H}_{-}^{n}$ ) and its orthogonal is integrable, therefore Theorem 2 of [9] implies that $\widetilde{M}$, the universal cover of $M$, is diffeomorphic to the product of the universal cover of a leaf of $\mathcal{G}^{\prime}$ by the universal cover of a leaf of $\mathcal{F}^{\prime}$. Moreover, we have seen that the function $\alpha$ vanishes only once on each leaf of $\mathcal{G}^{\prime}$. Consequently, $\alpha^{-1}(0)$ is connected and is equal to exactly one leaf of $\mathcal{F}^{\prime}$.

We denote by $\widetilde{g}, \widetilde{\alpha}, \widetilde{\mathcal{F}^{\prime}}$ and $\widetilde{\mathcal{G}^{\prime}}$ the lifts to $\widetilde{M}$ of $g, \alpha, \mathcal{F}^{\prime}$ and $\mathcal{G}^{\prime}$. Fact 4.14 and the proof of Lemma 4.13 imply that $\widetilde{M} \backslash \widetilde{\alpha}^{-1}(0)$ is diffeomorphic to $\mathbb{R}_{>0} \times S^{n-1} \times F$, where the factor $\mathbb{R}_{>0}$ corresponds to the direction of the vector field $\bar{X}$. Furthermore, according to Theorem 3.3, there exists a metric $h$ on $N=S^{n-1} \times F$ and a parallel tensor $S$ on $(N, h)$ such that the metric $\widetilde{g}$ reads $-d s^{2}+\sinh ^{2}(s)(h-S)+\cosh ^{2} S$. From the proof of Theorem 3.3, we deduce that $h-S$ is a metric on $S^{n-1}$, and $S$ is a metric on $F$, we denote these metrics respectively $-g_{S}$ and $g_{F}$. The term $-d s^{2}-\sinh ^{2}(s) g_{S}$ is the metric of $\mathbb{H}_{-}^{n}$ in polar coordinates, therefore $g_{S}$ is the canonical metric of the sphere. Hence, $(\widetilde{M}, \widetilde{g})$ is the warped product of $\mathbb{H}_{-}^{n}$ and $\left(F, g_{F}\right)$ by the function $f$ defined by $f(x)=\cosh ^{2}\left(d_{H}(O, x)\right)$, where $d_{H}$ is the hyperbolic distance and $O$ is a point of $\mathbb{H}_{-}^{n}$, therefore we denote it now $\mathbb{H}_{-}^{n} \times_{f} F$.

The manifold $M$ is the quotient of $\mathbb{H}_{-}^{n} \times_{f} F$ by the action of its fundamental group $\Pi$. This action preserves in particular the metric, the function $\widetilde{\alpha}$ and the foliations $\widetilde{\mathcal{G}}^{\prime}$ and $\widetilde{\mathcal{F}^{\prime}}$, therefore $\Pi \subset \operatorname{Isom}\left(\mathbb{H}_{-}^{n}\right) \times \operatorname{Diff}(F)$. 
We denote by $\Lambda$ the projection of $\Pi$ on $\operatorname{Diff}(F)$. As the leaves of $\mathcal{G}^{\prime}$ are always diffeomorphic to $\mathbb{R}^{n}$, any element of $\Pi$ fixing a point of $F$ has to be trivial. It means that there exists a morphism $\rho: \Lambda \rightarrow \operatorname{Isom}\left(\mathbb{H}_{-}^{n}\right)$ such that $\Pi=(\rho(\Lambda), \Lambda)$. Moreover, the action of $\Pi$ on $\widetilde{M}$ preserves $\widetilde{\alpha}^{-1}(0)$, therefore $\rho(\Lambda)$ fixes the point $O, \Lambda \subset \operatorname{Isom}\left(F, g_{F}\right)$ and $\Lambda$ acts freely and properly discontinuously on $F$ (and $F / \Lambda=\alpha^{-1}(0)$ ).

Conversely, let $f: \mathbb{H}_{-}^{n} \rightarrow \mathbb{R}$ defined as above and let $\left(F, g_{F}\right)$ be a pseudoRiemannian manifold. Clearly (see Examples 6.4 for more details), $\mathbb{H}_{-}^{n} \times_{f} F$ has a decomposable cone. Moreover, for any group $\Lambda$ acting freely, properly discontinuously and isometrically on $\left(F, g_{F}\right)$ and for any morphism $\rho: \Lambda \rightarrow$ $\operatorname{Isom}\left(\mathbb{H}_{-}^{n}\right)$ such that $\rho(\Lambda)$ fixes $O$, the quotient $\left(\mathbb{H}_{-}^{n} \times{ }_{f} F\right) /(\rho(\Lambda), \Lambda)$ is a well defined pseudo-Riemannian manifold. Finally, the parallel decomposition of the cone over $\mathbb{H}_{-}^{n} \times_{f} F$ being invariant by the action of $(\rho(\Lambda), \Lambda)$, it induces a parallel decomposition of the cone over $\left(\mathbb{H}_{-}^{n} \times_{f} F\right) /(\rho(\Lambda), \Lambda)$.

The last point to investigate is the geodesic completeness of $(M, g)$ and $\left(F, g_{F}\right)$. Our main tool is the following proposition.

Proposition 4.15 (see [8] Proposition 7.38 p. 208). A curve $\gamma=$ $\left(\gamma_{1}, \gamma_{2}\right)$ in $\mathbb{H}_{-}^{n} \times_{f} F$ is a geodesic if and only if

$$
\begin{aligned}
& \gamma_{1}^{\prime \prime}=h\left(\gamma_{2}^{\prime}, \gamma_{2}^{\prime}\right) f \circ \gamma_{1} \operatorname{grad} f, \\
& \gamma_{2}^{\prime \prime}=\frac{-2}{f \circ \gamma_{1}}\left(f \circ \gamma_{1}\right)^{\prime} \gamma_{2}^{\prime} .
\end{aligned}
$$

The point $O$ being a critical point of $f$, we deduce from Proposition 4.15 that $\{O\} \times F$ is totally geodesic. Hence, if $(M, g)$ is geodesically complete then $\left(F, g_{F}\right)$ is also complete.

Conversely, we suppose $\left(F, g_{F}\right)$ complete. Let $\gamma(t)=\left(\gamma_{1}(t), \gamma_{2}(t)\right)$ be a geodesic of $(M, g)$ and let $\Gamma_{2}$ be the locus of the geodesic of $\left(F, g_{F}\right)$ with initial speed $\gamma_{2}^{\prime}(0)$. It follows from Proposition 4.15 that $\mathbb{H}_{-}^{n} \times \Gamma_{2}$ is a totally geodesic submanifold of $(M, g)$ that contains $\gamma$. There are three cases to consider according to the type of $\gamma_{2}^{\prime}(0)$.

If $h\left(\gamma_{2}^{\prime}, \gamma_{2}^{\prime}\right)=0$, then $\gamma_{1}$ is a geodesic of $\mathbb{H}_{-}^{n}$. We write $\gamma_{2}(t)$ as $\gamma_{2}(u(t))$, where $u$ is a geodesic parameterization of $\Gamma_{2}$. We have

$$
u^{\prime \prime}(t)=\frac{-2\left(f \circ \gamma_{1}\right)^{\prime}(t)}{f \circ \gamma_{1}(t)} u^{\prime}(t)
$$

therefore $u^{\prime}(t)=C\left(f \circ \gamma_{1}\right)^{-2}(t)$. Moreover, $f \circ \gamma_{1} \rightarrow \infty$ implies $t \rightarrow \infty$. Hence the geodesic $\gamma$ is complete. 
If $h\left(\gamma_{2}^{\prime}, \gamma_{2}^{\prime}\right)<0$, then the restriction of $g$ to $\mathbb{H}_{-}^{n} \times \Gamma_{2}$ is negative Riemannian and is therefore complete (see [8] Lemma 7.40 p. 209).

If $h\left(\gamma_{2}^{\prime}, \gamma_{2}^{\prime}\right)>0$, then the restriction of $g$ to $\mathbb{H}_{-}^{n} \times \Gamma_{2}$ has constant curvature equal to 1 (see, for example, Corollary 2.3 of [1]). Moreover its signature is $(1, n)$ and it contains a codimension 1 foliation by hyperbolic spaces and a complete geodesic (the one above $O$ ) perpendicular to this foliation. It means that the universal cover of $\mathbb{H}_{-}^{n} \times \Gamma_{2}$ is the universal cover of the (negative) anti de Sitter space and is therefore complete.

As $\gamma \subset \mathbb{H}_{-}^{n} \times \Gamma_{2}$, we have proven that $\mathbb{H}_{-}^{n} \times_{f}\left(F, g_{F}\right)$ (and therefore $(M, g))$ is complete.

\section{Cones admitting a parallel nilpotent symmetric endomorphism field}

We denote by $\mathbb{R}^{p+1, q}$ the space $\mathbb{R}^{p+q+1}$ equipped with the standard pseudoEuclidean metric of signature $(p+1, q)$. We choose coordinates such that the metric reads $2 x_{1} x_{2}+\sum_{3 \leq i \leq p+2} x_{i}^{2}-\sum_{p+3 \leq j \leq p+q+1} x_{j}^{2}$. We consider the pseudo-sphere $S^{p, q}=\left\{x \in \mathbb{R}^{p+1, q} \mid\langle x, x\rangle=1\right\}$ endowed with its natural metric. The cone over $S^{p, q}$ is the open subspace of $\mathbb{R}^{p+1, q}$ given by $\left\{x \in \mathbb{R}^{p+1, q} \mid\right.$ $\langle x, x\rangle>0\}$, the vector field $\partial_{r}$ being $\langle x, x\rangle^{-1 / 2} x$.

Obviously, the functions on $\mathbb{R}^{p+1, q}$ having a non-trivial parallel Hessian are the polynomial functions of degree 2 . We choose $A(x)=x_{1}^{2}$. Its Hessian is defined by the 2-step nilpotent endomorphism $2 \widetilde{T}$ where $\widetilde{T}(u)=\widehat{g}\left(\partial_{2}, u\right) \partial_{2}$. The function $\alpha$ is just the restriction of $A$ to $S^{p, q}$. The idea is to deform the metric of $S^{p, q}$ in such a way that the function $\alpha$ remains a solution of the Obata equation.

It is clear that $\alpha^{-1}(0)$ is a codimension 1 totally geodesic lightlike submanifold. Actually, the level sets of $\alpha$ define a smooth codimension 1 foliation ( $\alpha$ is not a submersion but it has a square root, which is a submersion). Each connected component of $S^{p, q} \backslash \alpha^{-1}(0)$ is isometric to $\mathbb{R} \times \mathbb{R}^{p+q-1}$ endowed with the metric $g_{0}=-d t^{2}+e^{-2 t} h_{0}$, where $h_{0}$ is the usual flat metric of $\mathbb{R}^{p, q-1}$.

We keep the notation of Section 2.2 and denote by $2 r X$ the gradient of $\alpha$. According to the proof of Theorem 3.3, we can choose the isometry above in such a way that it sends the vector field $\partial_{t}$ to $\bar{X}=|\langle 2 r X, 2 r X\rangle|^{-1 / 2} 2 r X$. Clearly, we have $2 r X=2 x_{1} \partial_{2}-2 x_{1}^{2} \partial_{r}$. Hence, if $\gamma=\left(\gamma_{1}, \ldots, \gamma_{p+q+1}\right)$ is an integral curve of $2 r X$ such that $\gamma_{1}(0)=1$ and $\gamma_{2}(0)=c$, then we have

$$
\gamma_{1}(t)=(4 t+1)^{-1 / 2}
$$




$$
\gamma_{2}(t)=(2 t+c)(4 t+1)^{-1 / 2}
$$

Let $h_{1}$ be any perturbation with compact support $K$ of the metric $h_{0}$ on $\mathbb{R}^{p+q-1}$. We endow $S^{p, q} \backslash \alpha^{-1}(0)$ with the metric $g_{1}=-d t^{2}+\mathrm{e}^{-2 t} h_{1}$. The metrics $g_{0}$ and $g_{1}$ coincide outside the union of the integral curves of $\partial_{t}$, or equivalently of $2 r X$, starting from $\{0\} \times K$. It follows from (5.1) and (5.2) that this set is a closed subset of $S^{p, q}$ which does not meet $\alpha^{-1}(0)$. It means that there exists an open neighborhood $U$ of $\alpha^{-1}(0)$ such that the metrics $g_{0}$ and $g_{1}$ coincide on $U \backslash \alpha^{-1}(0)$. Consequently, the metric $g_{1}$ extends to a smooth metric $g$ on $S^{p, q}$.

It follows from Theorem 3.3 that the restriction of $\alpha$ is a solution of the Obata equation on the manifold $S^{p, q} \backslash \alpha^{-1}(0)$ endowed with the metric $g_{1}$. Thus, $\alpha$ is a solution of the Obata equation on an open dense subset of $\left(S^{p, q}, g\right)$ and therefore on the whole manifold.

We can construct this way a large family of pseudo-Riemannian manifolds with non-constant curvature whose cone admits a rank 1 parallel nilpotent symmetric endomorphism field. This absence of rigidity, that contrasts with the decomposable case, is related to the fact that the gradient lines of $\alpha$ never accumulate on critical points.

Let us look at the lacks of the examples we have constructed:

- We did not prove that $h_{1}$ can be chosen such that $g$ is geodesically complete. The main reason is that $g$ is clearly non-extendable, therefore, in some sense, complete enough.

- We have chosen a situation where the rank of $\widetilde{T}$ is 1 . It allowed us to perturb $h_{0}$ without thinking of $T$. If $\widetilde{S}$ is a parallel nilpotent symmetric endomorphism on a flat manifold $(N, h)$, it is possible to perturb $h$ while keeping the endomorphism $\widetilde{S}$ parallel. Hence, our construction could also provide examples with $\widetilde{T}$ of higher rank.

- The metric $g$ is still flat on a neighborhood of $\alpha^{-1}(0)$. This is perhaps the main problem. We did not start the discussion about all the admissible metrics $h_{1}$. For example, it seems reasonable to think that we can replace the metric $h_{1}$ by a metric which is asymptotically flat. In fact, we prefer to ask if there exist non-flat real analytic examples.

\section{Application to projective geometry}

We define the degree of mobility of a pseudo-Riemannian metric $g$ as the dimension of the space of metrics projectively (or geodesically) equivalent 
to $g$, i.e., the set of metrics having the same unparameterized geodesics as $g$. This number is well defined see [6] for details. It is always positive as the connection is invariant when $g$ is multiplied by a constant. We will say that two metrics are affinely equivalent if they have the same parameterized geodesics, i.e., if their Levi-Civita connections coincide.

Using results of [6], Matveev and the author proved in [7] the following result:

Theorem 6.1. Let $g$ be a pseudo-Riemannian metric on an $(n>1)$-dimensional closed connected manifold. Then, if the metric $\bar{g}$ on $M$ is geodesically equivalent to $g$, but not affinely equivalent to $g$, then the degree of mobility of $g$ is precisely 2 or there exists $c \neq 0$ such that $c g$ is Riemannian and has constant curvature equal to one.

This result is still true, with no hypotheses on the topology of $M$, if the metrics $g$ and $\bar{g}$ are assumed to be complete (see [6]). We will see that it is not the case if $g$ only is assumed to be complete. As we said in the introduction, there is a link between the Obata equation and projective geometry. In [6], it is proven that if the degree of mobility of a metric is greater than 2 then the Obata equation has a non-trivial solution. The following proposition (which is probably known) explicit the converse, i.e., how a geodesically equivalent metric $\bar{g}$ is obtained from a parallel tensor $T$ on $\widehat{M}$.

Proposition 6.2. If $(M, g)$ is a manifold such that its cone admits a nontrivial parallel symmetric 2-tensor field $T$ such that $T\left(\partial_{r}, \partial_{r}\right)$ is bounded from above or below then there exists a metric $\bar{g}$ on $M$ which is projectively equivalent to $g$ but not affinely equivalent to $g$.

Proof. Let $T$ be a non-trivial parallel symmetric 2-tensor field on $\widehat{M}$ such that $T\left(\partial_{r}, \partial_{r}\right)$ is bounded from above or below. As for any $(a, b) \in \mathbb{R}^{2}$ the tensor field $a T+b \widehat{g}$ is parallel, we can assume that $T$ is non-degenerate and $\alpha=T\left(\partial_{r}, \partial_{r}\right)$ is positive. Thus it is a pseudo-Riemannian metric. Its LeviCivita connection is the only torsion free connection $\nabla$ such that $\nabla T=0$. As $\widehat{D} T=0$, this connection is $\widehat{D}$.

We are going to change the parameterization of the factor $\mathbb{R}_{>0}$ in order to see that $T$ is also a cone metric. Let $\psi: M \rightarrow \widehat{M}$ defined by $\psi(m)=$ $\left(\alpha(m)^{-1 / 2}, m\right)$. It follows from Corollary 2.10 that the submanifold $\psi(M)$ is orthogonal to $\partial_{r}$ with respect to $T$.

According to Fact 2.2 , we have $\widehat{D} .\left(r \partial_{r}\right)=\mathrm{Id}$, therefore

$$
\left(\mathcal{L}_{r \partial_{r}} T\right)(u, v)=T\left(\widehat{D}_{u}\left(r \partial_{r}\right), v\right)+T\left(\widehat{D}_{v}\left(r \partial_{r}\right), u\right)=2 T(u, v) .
$$


We put $\rho=r \sqrt{\alpha}$, as $\rho \partial_{\rho}=r \partial_{r}$ the equation (6.1) implies that

$$
T=d \rho^{2}+\rho^{2} g^{\prime}
$$

where $g^{\prime}$ is the restriction of $T$ to $\psi(M)$. Thus, $T$ is the cone metric of $\left(\psi(M), g^{\prime}\right)$. A cone metric being a warped product, we have:

Fact 6.3 (see $[8]$ p. 208). The projection of a geodesic of $(\widehat{M}, \widehat{D})$ on $M$ (resp. on $\psi(M))$ is a pregeodesic of $(M, g)$ (resp. of $\left.\left(\psi(M), g^{\prime}\right)\right)$.

In order to obtain a metric on $M$, we put $\bar{g}=\psi^{*} g^{\prime}=\psi^{*} T$. The geodesics of $\left(\psi(M), g^{\prime}\right)$ being projected on the geodesics of $(M, \bar{g})$, we deduce from Fact 6.3 that the metrics $g$ and $\bar{g}$ are projectively equivalent. To see that they are not affinely equivalent, it is possible to compute explicitly the reparameterization of the geodesics. It can be done with the help of section 5 of [1], where the geodesic lift of a geodesic of $(M, g)$ to $\widehat{M}$ is explicitly computed (see also [6] section 2.4). There always exist geodesics that are not affinely reparameterized.

Examples 6.4. 1. Let $M=S^{p, q}=\left\{x \in \mathbb{R}^{p+1, q} \mid\langle x, x\rangle=1\right\}$. We have already seen in Section 5 that $\widehat{M}=\left\{x \in \mathbb{R}^{p+1, q} \mid\langle x, x\rangle>0\right\}$. The tensor $T$ considered in the proof of Proposition 6.2 is the restriction to $\widehat{M}$ of a non-degenerate bilinear form on $\mathbb{R}^{p+q+1}$ such that the radial vector field of $\mathbb{R}^{p+q+1}$ is never lightlike with respect to $T$. Moreover $\psi(M)=$ $\{x \in \widehat{M} \mid T(x, x)=1\}$, i.e., it is the intersection of the (pseudo)sphere of $T$ and $\widehat{M}$. Eventually, the metric $\bar{g}$ is the pull-back of $T_{\mid \psi(M)}$ on $M=S^{p, q}$. When $M$ is the round sphere and $T$ is positive definite, we obtain the classical Beltrami examples of geodesically equivalent spheres. If $T$ and $\widehat{g}$ are neither both Riemannian nor proportional, then $\psi(M)$ is a proper open subset of the (pseudo)sphere of $T$ and therefore $\bar{g}$ is not complete.

2. Let $(M, g)=\mathbb{H}_{-}^{n} \times_{f} F$ be one of the manifolds obtained in Theorem 4.11. We see $\mathbb{H}_{-}^{n}$ as a connected component of $S^{1, n}$ and we choose coordinates $\left(x_{0}, x_{1}, \ldots, x_{n}\right)$ on $\mathbb{R}^{1, n}$ such that the metric reads $d x_{0}^{2}-$ $\sum_{1 \leq i \leq n} d x_{i}^{2}$. The cone $\widehat{M}$ is $\left\{x \in \mathbb{R}^{1, n} \mid\langle x, x\rangle>0, x_{0}>0\right\} \times F$ and the metric $\widehat{g}$ reads $d x_{0}^{2}-\sum_{1 \leq i \leq n} d x_{i}^{2}+x_{0}^{2} g_{F}$. The parallel $n$-dimensional flat distribution of $(\widehat{M}, \widehat{g})$ is clearly $\operatorname{Span}\left(\partial_{x_{1}}, \ldots, \partial_{x_{n}}\right)$. Let $\widetilde{T}_{0}$ be the projection on this distribution and $\widetilde{T}=\mathrm{Id}-2 \widetilde{T}_{0}$. The 2-tensor field $T$ reads $d x_{0}^{2}+\sum_{1 \leq i \leq n} d x_{i}^{2}+x_{0}^{2} g_{F}$. 
Using polar coordinates on $\mathbb{H}_{-}^{n}$, the function $\psi$ defined in the proof of Proposition 6.2 can be seen as a function from $\mathbb{R}_{>0} \times S^{n-1} \times F$ to $\mathbb{R}^{1, n} \times F$. Using the coordinates introduced above on $\mathbb{R}^{1, n}$ and seeing $S^{n-1}$ as a submanifold of $\mathbb{R}^{n}$, it reads

$\psi(s, p, x)=\left(\frac{\cosh (s)}{\sqrt{\cosh ^{2}(s)+\sinh ^{2}(s)}}, \frac{\sinh (s)}{\sqrt{\cosh ^{2}(s)+\sinh ^{2}(s)}} p, x\right)$.

It follows that

$\psi^{*} T=\bar{g}=\xi(s) d s^{2}+\frac{\sinh ^{2}(s)}{\cosh ^{2}(s)+\sinh ^{2}(s)} g_{S}+\frac{\cosh ^{2}(s)}{\cosh ^{2}(s)+\sinh ^{2}(s)} g_{F}$,

where $\xi(s)=\left(\sinh ^{2}(s)+n \cosh ^{2}(s)\right)\left(\cosh ^{2}(s)+\sinh ^{2}(s)\right)^{-3}$ and $g_{S}$ is the standard metric on the sphere. The fact that $\xi$ is not constant shows that $g$ and $\bar{g}$ are not affinely equivalent. As $\int_{0}^{+\infty} \xi(s)^{1 / 2} d s<\infty$, the metric $\bar{g}$ is not geodesically complete.

Moreover, if $n>1$, the distribution $\operatorname{Span}\left(\partial_{x_{1}}, \ldots, \partial_{x_{n-1}}\right)$ is another parallel distribution of $\widehat{M}$. We can repeat the construction above with the projection on this distribution in place of $\widetilde{T}_{0}$ and produce another geodesically equivalent metric. Actually, it is possible to do the construction with almost any linear combination of these projections, consequently the degree of mobility of $g$ is greater than 2 .

Let us remark that, as there exists a $(n-1)$-dimensional parallel distribution on $\widehat{M}$, Theorem 4.11 says that $(M, g)$ is also the warped product of $\mathbb{H}_{-}^{n-1}$ and a pseudo-Riemannian manifold $\left(F^{\prime}, g_{F^{\prime}}\right)$. It is not difficult to see that $\left(F^{\prime}, g_{F^{\prime}}\right)=\left(\mathbb{R} \times F,-d t^{2}+g_{F}\right)$.

3. We could also use the example of Section 5, to obtain a more exotic family of projectively equivalent metrics with non-constant curvature. But in this case, the degree of mobility is probably equal to 2.

We have proven in point 2 of Examples 6.4:

Corollary 6.5. There exist pseudo-Riemannian metrics $g$ and $\bar{g}$ both with non-constant curvature on a manifold $M$ such that

1. $g$ is geodesically complete,

2. $g$ and $\bar{g}$ are projectively equivalent but not affinely equivalent,

3. the degree of mobility of $g$ and $\bar{g}$ is greater than 2 . 
It seems natural to wonder now if the metrics above are the only complete pseudo-Riemannian manifolds with non-constant curvature and a degree of mobility greater than 2 that admit projectively but not affinely equivalent metrics. In particular, we can ask if there exist complete pseudo-Riemannian manifolds with non-constant curvature whose cone admits a higher order nilpotent endomorphism.

\section{References}

[1] D.V. Alekseevsky, V. Cortés, A.S. Galaev and T. Leistner, Cones over pseudo-Riemannian manifolds and their holonomy, J. Reine Angew Math. 635 (2009), 23-69.

[2] C. Bär, Real Killing spinors and holonomy, Commun. Math. Phys. 154(3) (1993), 509-521.

[3] C.P. Boyer and K. Galicki, Sasakian geometry, in Oxford Mathematical Monographs, Oxford University Press, Oxford, 2008. xii+613 pp.

[4] C. Bohle, Killing spinors on Lorentzian manifolds, J. Geom. Phys. 45(3-4) (2003), 285-308.

[5] S. Gallot, Équations différentielles caractéristiques de la sphère, Ann. scient. Éc. Norm. Sup. $4^{e}$ série t.12, 1979, 235-267.

[6] V. Kiosak and V. Matveev, Proof of projective Lichnerowicz conjecture for pseudo-Riemannian metrics with degree of mobility greater than two, Commun. Math. Phys. 297(2) (2010), 401-426.

[7] V. Matveev and P. Mounoud, Gallot-Tanno theorem for closed incomplete pseudo-Riemannian manifolds and applications, Ann. Global Anal. Geom. 38(3) (2010), 259-271.

[8] B. O’Neill, Semi-Riemannian geometry, with application to relativity, Academic Press, 1983.

[9] R. Ponge and H. Reckziegel, Twisted products in pseudo-Riemaniann geometry, Geom. Dedicatae 48 (1993), 15-25.

[10] A.S. Solodovnikov, Projective transformations of Riemannian spaces, Uspehi Mat. Nauk (N.S.) 11(470) (1956), 45-116.

[11] S. Tanno, Some differential equations on Riemannian manifolds, J. Math. Soc. Japan 30(3) (1978), 509-531. 
Univ. Bordeaux, IMB, UMR 5251

F-33400 Talence, France

E-mail address: Pierre.Mounoud@math.u-bordeaux1.fr

Received November 9, 2010 
